\title{
Perspectives on pathogenesis and prevention of congenital herpes virus infection Mark R Schleiss
}

\author{
Address: Pediatric Infectious Diseases, University of Minnesota Medical School and Center for Infectious Diseases and Microbiology Translational \\ Research, Minneapolis, MN, USA \\ from Fifth Dominique Dormont International Conference. Mother-to-child transmitted viral diseases: from transmission to children care \\ Paris, France. 26-28 March 2009 \\ Published: 22 July 2009 \\ Retrovirology 2009, 6(SuppI I):L2 doi:I0.I I86/I742-4690-6-SI-L2
}

This abstract is available from: http://www.retrovirology.com/content/6/SI/L2

(c) 2009 Schleiss; licensee BioMed Central Ltd.

Each of the 8 members of the human herpesvirus family identified to date has been associated, to varying degrees, with congenital and perinatal transmission to the fetus and newborn infant. The problem of prevention of maternal-to-child transmission of herpesvirus infections is complicated by a lack of understanding of correlates of protective immunity for both the mother and the fetus. Herpesviruses encode multiple immune evasion genes, and establish lifelong, persistent infection in the host. These issues render the design of protective vaccines highly problematic.

In spite of these challenges, progress has been made in recent years toward the development of new vaccines for herpesviruses, in particular the viruses that pose the greatest risk to the newborn - herpes simplex virus (HSV) and cytomegalovirus (CMV). Recent strategies undergoing evaluation in clinical trials include both the approach of attenuated, live-virus vaccines as well as recombinant, subunit vaccines that target immunodominant virallyencoded proteins. A recombinant subunit vaccine against genital HSV infection, based on the viral glycoprotein gD, has demonstrated efficacy in placebo-controlled studies, and merits further investigation. A benefit of immunization against genital HSV for prevention of neonatal HSV infection is inferred, although this endpoint may ultimately be difficult to demonstrate in clinical trials. Progress has also been made for immunization against congenital infection with CMV. Given the neurodevelopmental injury associated with congenital CMV infection, such a vaccine is a major public health priority. Recently, subunit vaccination with the $\mathrm{CMV}$ glycoprotein, $\mathrm{gB}$, has demonstrated efficacy against acquisition of infection in a phase II trial in young women. In a placebo-controlled study, vaccination had a significant impact on the proba- bility of a study participant remaining CMV seronegative through the 42-month follow-up period. These data are the first that demonstrate significant efficacy of a CMV vaccine for prevention of infection, and will drive interest in future $\mathrm{gB}$ vaccine studies using other expression technologies. Although these results are encouraging, ultimately, several major issues must be resolved before CMV vaccines can be optimized. First, the phenomena of reinfection of CMV-immune hosts with new strains must be understood, since non-primary CMV infections account for the major disease burden of congenital CMV. Presumably, vaccine design will need to account for the heterogeneity in clinical isolates that may account for most reinfections. Secondly, the role of viral immune evasion genes in abrogating host immunity must be clarified: a better understanding of this process may, in turn, allow design of optimized live, attenuated vaccines. Finally, the role of immune responses that may potentially control infection at mucosal surfaces needs to be elucidated, and this knowledge may in turn facilitate development of novel vaccines that block primary infection at mucosal sites. 